\title{
Body Height and Its Assessment By Using the Arm Span of Adolescents From the Montenegrin Municipalities of Berane and Pljevlja
}

\author{
Marija Bubanja'1 \\ 'University of Montenegro, Faculty for Sport and Physical Education, Niksic, Montenegro
}

\begin{abstract}
The purpose of this research was to examine Montenegrin adolescents' body height from the municipalities of Berane and Pljevlja, Montenegro. Also, the research examines an arm span as an alternative assessment for measuring the body height which varies from region to region in Montenegro. The research surveyed 275 adolescents (130 males and 145 females) from the previously mentioned towns in Montenegro. Anthropometric measurements were performed according to the protocol of the International Society for the Advancement of Kinanthropometry (ISAK). The final outcome of the research are mean values and standard deviations related to anthropometric measurements. The relationship between the body height and the arm span was determined by using simple correlation coefficients and their $95 \%$ confidence interval. This was followed by a linear regression analysis which was utilized to determine to what extent the arm span can reliably predict the body height. The results show that adolescents from the north part of Montenegro are high, men have an average body height of $182.67 \pm 6.08$ centimeters and the arm span of $183.02 \pm 6.71$ centimeters, while the average height of the female population is $169.46 \pm 5.96$ centimeters with a arm span of $168.56 \pm 7.30$ centimeters. Compared to other studies, the results of this research have shown that this population is taller than most nations throughout Europe. Moreover, as it was expected, the arm span can predict reliably the body height when it comes to this population.
\end{abstract}

Key words: Body Height, Arm Span, Anthropometry, Adolescents, Montenegro

\section{Uvod}

Postoji veliki broj studija koji potvrđuje da je procjena tjelesne visine značajan faktor koji može uticati na procjenu statusa uhranjenosti kod odraslih ljudi (Arifi, Bjelica, Sermaxhaj, Gardasevic, Kezunovic, \& Popovic, 2017a; Datta Banik, 2011; Popovic i Bjelica, 2016), ali i na procjenu razvoja djece, zatim na ocjenu osnovnih energetskih potreba, prilagođavanje mjera fizičkog kapaciteta, kao i procjenu količine uzimanja određenih ljekova i niza drugi stvari, kao što je procjena mišićne snage, mataboličkih procesa, kapaciteta pluća i sl. (Golshan, Amra, i Hoghogi, 2003; Golshan, Crapo, Amra, Jensen i Golshan, 2007; Mohanty, Babu, i Nair, 2001; Ter Goon, Toriola, Musa i Akusu, 2011; Popovic, Arifi, i Bjelica, 2017a; Popovic, i Bjelica, 2017). Međutim, prema Quanjeru i sar. (2014) tačna tjelesna visina se ne može uvijek identifikovati i riješiti na uobičajeni način (npr. paraliza, frakture, amputacija, skolioza i bol). Zbog ovih faktora procjena tjelesne visine se može dobiti na osnovu drugih pouzdanih antropometrijskih pokazatelja kao što su: dužina ruku i stopala, dužina potkoljenice, visina koljena, dužina podlaktice, dužina grudne kosti, sjedeća visina, dužina lopatice, raspon ruku, itd. (Gardasevic, Rasidagic, Krivokapic, Corluka, i Bjelica, 2017; Popovic, 2017; Popovic, Gardasevic, Masanovic, Arifi, i Bjelica, 2017; Masanovic, Gardasevic, i Arifi, 2018a; Masanovic, Gardasevic, i Arifi, 2018b; Gardasevic, 2018b; Arifi, Sermaxhaj, Gardasevic, Alaj, \& Metaj, 2018; Gardasevic, Masanovic, \& Arifi, 2018c; Arifi,

Correspondence:

Montenegro M. Bubanja

Gport University of Montenegro, Faculty for Sport and Physical Education, Narodne omladine bb, 81400 Niksic, Montenegro E-Mail: marijabubanja@ymail.com 
Gardasevic, \& Masanovic, 2018). Prema tome, svi ovi antropometrijski pokazatelji koji se koriste kao alternativa za procjenu stojeće visine tijela su vrlo važni za predviđanje gubitka visine tijela vezane za starenje. Dijagnostikovanje nesrazmjernog rasta i skeletne displazije, stanje gubitka visine tokom operativnih zahvata na kičmi (Mohanty i sar., 2001), kao i predviđanje stojeće visine kod mnogih starijih osoba, je vrlo teško izmjeriti precizno, a ponekad i nemoguće, zbog problema pokretljivosti i kifoze (Hickson i Frost, 2003). Važno je navesti da poznavanje ove oblasti pronalazi svoju važnost u sportskoj nauci, jer tjelesna visina predstavlja značajan faktor koji utiče na uspjeh u raznim sportskim disciplinama (Popovic, Bjelica, Petkovic, i Muratovic, 2012; Popovic, Milašinovic, Matic, Gardasevic, Bjelica, 2016). Neki autori smatraju da se odnos dugih kostiju i visine tijela razlikuje u različitim etničkim i rasnim grupama (Bjelica i sar., 2012; Brown, Feng, i Knapp, 2002; Gardasevic, 2018; Gardasevic, 2018a; Gardasevic, Masanovic, i Arifi, 2018; Gardasevic, Masanovic, i Arifi, 2018a; Gardasevic, Masanovic, i Arifi, 2018b; Masanovic, 2017; Masanovic, 2018; Masanovic, 2018a; Masanovic, Gardasevic, i Arifi, 2018e; Masanovic, Gardasevic, i Arifi, 2018f; Masanovic, Gardasevic, i Arifi, 2018g; Popovic, Bjelica, Molnar, Jakšić, i Akpinar, 2013; Popovic, Bjelica, Tanase, i Milasinovic, 2015; Reeves, Varakamin, i Henry, 1996; Steele i Chenier, 1990) kao i raznim regijama (Arifi, 2017; Arifi i sar., 2017b; Milasinovic, Popovic, Matic, Gardasevic, i Bjelica, 2016a; Milasinovic, Popovic, Jaksic, Vasiljevic, i Bjelica, 2016b; Masanovic, Gardasevic, i Arifi, 2018c; Masanovic, Gardasevic, i Arifi, 2018d; Bubanja, 2018). Autori su vjerovali da je populacija koja živi u sjevernoj regiji možda viša od prosječnih Dinarskih Alpa i viša od stanovništva iz centralne i južne regije (Bubanja, Vujović, Tanase, Hadzic, i Milasinovic, 2015; Milasinovic, Popovic, Jaksic, Vasiljevic i Bjelica, 2016a; Milasinovic, Gardasevic, i Bjelica, 2017) uglavnom zbog pretpostavke specifičnih fizičkih struktura karakterističnih za ljude iz sjevernog dijela Crne Gore.

Da su tjelesna visina i raspon ruku međusobno povezani, ukazuju podaci koji su dobijeni u istraživanjima središnje crnogorske regije (Vujovic i sar., 2015) i južne regije
(Milasinović, Popović, Matic, Gardasevic, i Bjelica, 2016a; Milasinovic, Popovic, Jaksic, Vasiljevic, i Bjelica, 2016b), Cilj ovog istraživanja bio je ispitati visinu tijela kod adolescenata u crnogorskim gradovima Pljevljima i Beranama, kao i ispitati odnos između visine tijela i raspona ruku kao alternative mjere za procjenu visine tijela.

\section{Metod}

Uzorak ispitanika u ovom istraživanju predstavlja 275 adoloscenata (130 muškaraca, 145 djevojaka) iz crnogorskih opština Berana i Pljevalja koji pohađaju završnu godinu srednje škole. Ova grupa je izabrana zato što rast pojedinca prestaje u ovom uzrastu i nema gubitka tjelesne težine. Prosječna starost ispitanika bila je $17.96 \pm 0.44$ godine (raspon od 17 do 19 godina).

Prema Marfell-Jones, Olds, Stewart, i Carter (2006), antropometrijska mjerenja, uključujući visinu tijela i raspon ruku, uzeti su u skladu sa protokolom Međunarodnog društva za unapređenje kinantropometrije (ISAK). Starost pojedinaca određena je direktno iz njihovog prijavljenog datuma rođenja.

Analiza je izvršena korišćenjem statističkog paketa za društvene nauke (SPSS) verzija 20.0. Aritmetička sredina i stadardna devijacija (SD) su izračunate za obije antropometrijske varijable. Odnos između visine tijela i raspona ruku utvrđen je korištenjem jednostavnih koeficijenata korelacije i njihovog $95 \%$ intervala pouzdanosti. Zatim je izvršena linearna regresiona analiza kako bi se utvrdilo u kojoj mjeri raspon ruku može pouzdano predvidjeti visinu tijela sa statističkom značajnošću od $\mathrm{p}<0.05$.

\section{Rezultati}

Rezultati antropometrijskih mjerenja za oba pola prikazani su u tabeli 1. Prosječna visina tijela za muškarce je 182.67 \pm 6.08 centimetara, rangirana sa minimalnim i maksimlnim rezultatima od 164.3-196.0 centimetara. Za djevojke prosjek visine tijela iznosi $169.46 \pm 5.96$ centimetara, rangiran sa minimalnim i maksimalnim rezultatom od $155.5-183.3$ centimetara. Prosječna vrijednost raspona ruku za muškarce je 183.02 \pm 6.71

Tabela 1. Antropometrijska parametri adoloscenata

\begin{tabular}{ccc}
\hline \multirow{2}{*}{ Pol } & $\begin{array}{c}\text { Visina Tijela } \\
\text { (Mean } \pm \text { SD) }\end{array}$ & $\begin{array}{c}\text { Raspon Ruku } \\
\text { (Mean } \pm \text { SD) }\end{array}$ \\
\hline \multirow{2}{*}{ Muški } & $164.3-196.0$ & $166.4-198.9$ \\
& $(182.67 \pm 6.08)$ & $(183.02 \pm 6.71)$ \\
\multirow{2}{*}{ Ženski } & $155.5-183.3$ & $147.8-188.3$ \\
& $(169.46 \pm 5.96)$ & $(168.56 \pm 7.30)$ \\
\hline
\end{tabular}

centimetara, sa minimalnim i maksimalnim rezultatom od 166.4 - 198.9 centimetara, dok je prosječna vrijednost raspona ruku kod djevojaka bila $168.56 \pm 7.30$ centimetara, rangirana sa minimalnim i maksimalnim rezultatom od 147.8-188.3 centimetara.
Koeficijent korelacije i njihova 95\%-na analiza intervala pouzdanosti između antropometrijskih mjerenja prikazani su u tabeli 2. Odnos između visine tijela i raspona ruku za oba pola bio je signifikantan $(\mathrm{p}<0.000)$, s koeficijentima korelacije (muškarci 0.802, djevojke 0.846).

Tabela 2. Korelacija između visine tijela i raspona ruku

\begin{tabular}{cccc}
\hline Pol & $\begin{array}{c}\text { Koeficijent } \\
\text { Korelecije }\end{array}$ & $\begin{array}{c}\text { 95\% Interval } \\
\text { Pouzdanosti }\end{array}$ & $\begin{array}{c}\text { Sig. } \\
\text { p-value }\end{array}$ \\
\hline Muški & 0.802 & $0.737-0.864$ & $<0.000$ \\
Ženski & 0.846 & $0.801-0.875$ & $<0.000$ \\
\hline
\end{tabular}

$\mathrm{U}$ tabeli 3. prikazani su rezultati linearne regresije u kojima su vidimo visoke vrijednosti koeficijenta regresije. To ukazuje na pozitivan odnos (muškarci 0.802, djevojke 0.846) koji dalje objašnjava da dužina raspona ruku značajno predviđa visinu tijela kod adolescentske populacije iz crnogorskih gradova Berana i Pljevalja (muškarci t $=15.167, \mathrm{p}<0.000$; djevojke 
Tabela 3. Rezultati linearne regresione analize

\begin{tabular}{cccccc}
\hline Pol & $\begin{array}{c}\text { Regresioni } \\
\text { Koeficijent }\end{array}$ & $\begin{array}{c}\text { Standardna } \\
\text { Greška (SE) }\end{array}$ & $\begin{array}{c}\text { Koeficijent } \\
\text { Determinacije(\%) }\end{array}$ & t-value & p-value \\
\hline Muški & 0.802 & 4.029 & 0.642 & 15.167 & 0.000 \\
Ženski & 0.846 & 3.908 & 0.716 & 18.982 & 0.000 \\
\hline
\end{tabular}

$\mathrm{t}=18.982, \mathrm{p}<0.000)$, što potvrđuje koeficijent determinacije za muškarce 0.642 i djevojke 0.716 . Prvi od svih modela izveden je uključivanjem starosti kao kovarijate. Međutim, utvrđeno je da je doprinos starosti bio beznačajan i stoga je starosna dob bila odbačena, a procjena izvedena kao univarijantna analiza. Visoke vrijednosti regresionog koeficijenta znače da raspon ruku značajno predviđa visinu tijela kod oba pola crnogorskih adolescenata iz pomenutih gradova.

Odnos između mjerenja raspona ruku i visine tijela među gore navedenim modelima je prikazan kao dijagram raspršivanja (Slika 1).

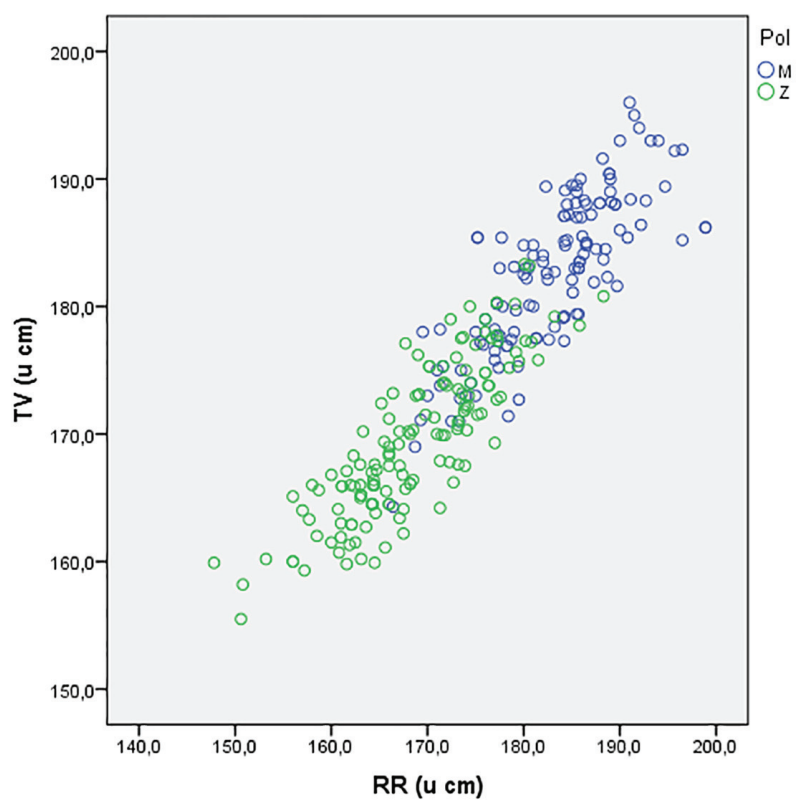

Slika 1. Dijagram raspršivanja i odnos između raspona ruku i visine tijela

\section{Diskusija}

Procjena tjelesne visine je mjera koja je vrlo često proučavana, dok je važno je istaknuti da je raspon ruku dobijen kao najpouzdaniji indikator tijela za predviđanje tjelesne visine pojedinca (Mohanty i sar., 2001; Ter Goon i sar., 2011).

Rezultati ove studije pokazali su da su adolescenti iz crnogorskih gradova Berana i Pljevalja vrlo visoki, u prosjeku 182.67 centimetara muškarci i 169.46 centimetara djevojke. To je nešto manje od prosjeka opšteg muškog stanovništva sa 183.21 centimetara i nešto viši od prosjeka opšteg ženskog stanovništva sa 168.37 centimetara u Crnoj Gori (Bjelica i sar., 2012). Na osnovu rezultata ove studije može se reći da je žensko stanovništvo najviše u Crnoj Gori, što je zaključeno poređenjem sa rezultatima prosječne tjelesne visine ženskog stanovništva centralne regije sa 169.24 centimetara (Bubanja i sar., 2015) i južne regije Crne Gore sa 168.73 centimetara (Milašinović, Popović, Jakšić, Vasiljević, i Bjelica, 2016). Prosječna visina muške populacije iz sjeverne regije Crne Gore je manja od populacije iz centralne regije 183.24 centimetra (Vujović, Bubanja, Tanase, i Vujović, 2015). Pineau i sar. (2005) su u svom istraživanju dokazali da je, suprotno opštem vjerovanju, žensko stanovništvo u Dinarskim Alpima, sa prosječnom visinom od 171 centimetra, približno drugoj po visini tijela populaciji u Holandiji, dok je muška populacija Dinarskih Alpa u prosjeku najviša u Evropi. Uzorak od 800 crnogorskih muškaraca koje je izmjerio Robert W. Ehrich (Coon, 1975) početkom 20. vijeka davao je najveći prosjek u cijeloj Evropi 177 cm, dok su se neke oblasti približavale 178 centimetara. Stoga je ova studija izazvala mnoge naučnike da vjeruju da bi crnogorska populacija mogla biti najviša populacija u Evropi. Međutim, Bjelica i njegovi saradnici (2012) potvrđuju da je ženska populacija visoka, ali ne i najviša sa 168,37 centimetara, dok je i muška populacija takođe visoka, ali ne i najviša sa 183,2 centimetra. Novije istraživanje sprovedeno kao nacionalno istraživanje (Popović, 2016; Popović, Bjelica i Hadžić, 2014; Popović, Bjelica, Milašinović, i Gardašević, 2016;) gdje su uzorak ispitanika predstavljali učenici završne godine srednje škole potvrdili su rezultate prethodne studije i ustanovili da je prosječna visina muške populacije u Crnoj Gori bila 183.74 centimetra. Međutim, postoji hipoteza da stanovnici Crne Gore još uvijek nijesu ostvarili svoj puni genetski potencijal jer su u poslednjih nekoliko decenija bili pod uticajem različitih faktora životne sredine, kao što su ratovi, loša ekonomska situacija itd. (Popović, Bjelica, Jakšić, i Akpinar, 2013). Dakle, autori smatraju da su te okolnosti imale negativan uticaj na sekularni trend u sjevernoj Crnoj Gori, dok se očekuje da će sekularne promjene koje utiču na visinu tijela u narednih 20 godina porasti, upoređujući ih sa razvijenim zemljama u kojima je ovaj trend već završen, kao što je Holandija (Schonbeck i sar., 2013).

$\mathrm{Na}$ kraju, možemo zaključiti, da raspon ruku pouzdano predviđa visinu tijela, što se može vidjeti na osnovu postignutih rezultata dobijenih pomoću linearne regresione analize gdje je statistički značajnost bila $\mathrm{p}<0.00$. Odnos između 
visine tijela i raspona ruku provjeren je pomoću Pearsonove korelacijske analize sa pouzdanošću od $95 \%$ kod muškaraca i djevojaka koji su imali vrlo visoku vrijednost (0.802 i 0.846$)$ međusobnih korelacija. Ovo upućuje na potrebu ispitivanja tjelesne visine $\mathrm{u}$ ostalim gradovima u Crnoj Gori kako bi se konsolidiralo studijsko područje Dinarskih Alpa.

\section{Acknowledgements}

There are no acknowledgements.

\section{Conflict of Interest}

The authors declare that there are no conflicts of interest.

Received: 10 May 2018 | Accepted: 13 July 2018 | Published: 29 October 2018

\section{References}

Arifi, F. (2017). Stature and its estimation utilizing arm span measurements of both gender adolescents from southern region in Kosovo. Sport Science, 10(1), 92-5.

Arifi, F., Bjelica, D., Sermaxhaj, S., Gardasevic, J., Kezunovic, M., \& Popovic, S. (2017a). Stature and its Estimation Utilizing Arm Span Measurements in Kosovan Adults: National Survey. International Journal of Morphology, 35(3):1161-7.

Arifi, F., Gardasevic, J., \& Masanovic, B. (2018). Relationship between foot length measurements and body height: A prospective regional study among adolescents in central region of Kosovo. Sport Mont, 16(3), 7579. doi: $10.26773 / \mathrm{smj} .181013$

Arifi, F., Sermaxhaj, S., Gardasevic, J., Alaj, I., \& Metaj, Z. (2018). Stature and Its Estimation Utilizing Arm Span Measurements of both gender Adolescents from Southern Region in Kosovo. Sport Mont, 16(2), 51-54. doi: 10.26773/smj.180609

Bjelica, D., Popovic, S., Kezunovic, M., Petkovic, J., Jurak, G., \& Grasgruber P. (2012). Body Height and Its Estimation Utilizing Arm Span Measurements in Montenegrin Adults. Anthropological Notebooks, 18(2), 69-83.

Bubanja, M., Vujovic, D., Tanase, G. D., Hadzic, R., \& Milasinovic, R. (2015) Body Height and Its Estimation Utilizing Arm Span Measurements in Female Adolescents from Central Region in Montenegro. Sport Mont, 12(43-45), 277-82

Bubanja, M. (2018). Body Height and Its Estimation Utilizing Arm Span Measurements in Adolescents from Northern Region in Montenegro. Journal of Anthropology of Sport and Physical Education, 2(3), 67-71.

Brown, J.K., Feng, J.Y., \& Knapp, T.R. (2002). Is self-reported height or arm span a more accurate alternative measure of height? Clinical Nursing Research, 11(4), 417-32.

Coon, C. (1975). The races of Europe. Westport, Conn: Greenwood Press.

Datta Banik, S. (2011). Arm span as a proxy measure for height and estimation of nutritional status: A study among Dhimals of Darjeeling in West Bengal India. Annals of Human Biology, 38(6), 728-35.

Gardasevic, J. (2018). Standing Height/Tibia Length Ration in WesternKosovan Adolescents. Retrieved from SSRN's eLibrary: https://ssrn. com/abstract $=3138101$

Gardasevic, J. (2018a). Standing Height/Sitting Height Ration in EasternKosovan Adolescents. Retrieved from SSRN's eLibrary: https://ssrn. com/abstract $=3141566$

Golshan, M., Amra, B., \& Hoghogi, M. A. (2003). Is arm span an accurate measure of height to predict pulmonary function parameters? Monald Arch. Chest, Disease 59(3), 189-92.

Gardasevic, J. (2018b). Relationship between Sitting Height Measurements and Standing Height: A Prospective Regional Study among Adolescents in Eastern Region of Kosovo. Sport Mont, 16(2), 15-19. doi: 10.26773/ smj. 180603

Gardasevic, J., Masanovic, B., Arifi, F. (2018). Relationship Tibia Length/ Standing Height in Central-Kosovan Adolescents. Retrieved from SSRN's eLibrary: https://ssrn.com/abstract=3138122

Gardasevic, J., Masanovic, B., Arifi, F. (2018a). Relationship Tibia Length/ Standing Height in Northern-Kosovan Adolescents. Retrieved from SSRN's eLibrary: https://ssrn.com/abstract=3138112

Gardasevic, J., Masanovic, B., Arifi, F. (2018b). Relationship Tibia Length/ Standing Height in Southern-Kosovan Adolescents. Retrieved from SSRN's eLibrary: https://ssrn.com/abstract=3138105

Gardasevic, J., Masanovic, B., \& Arifi, F. (2018c). Relationship between tibia length measurements and standing height: A prospective regiona study among adolescents in southern region of Kosovo. Sport Mont, 16(3), 51-55. doi: 10.26773/smj.181009

Gardasevic, J., Rasidagic, F., Krivokapic, D., Corluka, M., \& Bjelica, D. (2017)
Stature and Its Estimation Utilizing Arm Span Measurements in Male Adolescents from Herzeg-Bosnia Entity in Bosnia and Herzegovina. Montenegrin Journal of Sports Science and Medicine, 6(1), 37-44.

Golhan, M., Amra, B., Hoghoghi, M.A. (2003). Is arm span an accurate measure of height to predict pulmonary function parameters? Monaldi Arch. Chest Dis., 59(3), 189-92.

Golshan, M., Crapo, R.O., Amra, B., Jensen, R.I., \& Golshan, R. (2007). Arm span as an independent predictor of pulmonary function parameters: validation and reference values. Respirology, 12(3), 361-6.

Hickson, M., \& Frost, G.A (2003). Comparison of three methods for estimating height in the acutely ill elderly population. Journal of Human Nutrition and Dietitian, 16(1), 13-20.

Masanovic, B. (2017). Relationship between arm span measurements and body height in Dinaric Alpes population: A systematic review. Journal of Anthropology of Sport and Physical Education, 1(1), 33-7.

Masanovic, B. (2018). Standing Height and its Estimation Utilizing Arm Spam and Foot Length Measurements in Dinaric Alps Population: A Systematic Review. Sport Mont, 16(2), 101-6. doi: 10.26773/smj.180619

Masanovic, B. (2018a). Standing Height/Sitting Height Relationhip in Western Region in Kosovo. Retrieved from SSRN's eLibrary: https://ssrn com/abstract $=3138518$

Masanovic, B. (2018b). Tibia Length and Standing Heigh Relationshipt in Eastern Region of Kosovo. Retrieved from SSRN's eLibrary: https://ssrn. com/abstract $=3143118$

Masanovic, B., Gardasevic, J., \& Arifi, F. (2018a). Relationship between foot length measurements and body height: A prospective regional study among adolescents in eastern region of Kosovo. Sport Mont, 16(1), 9-13. doi: $10.26773 / \mathrm{smj} .180202$

Masanovic, B., Gardasevic, J., \& Arifi, F. (2018b). Relationship between Foot Length Measurements and Body Height: A Prospective Regional Study among Adolescents in Northern Region of Kosovo. AnthropologieInternational Journal of Human Diversity and Evolution, in pres, https:// doi.org/10.26720/anthro.18.01.23.1.

Masanovic, B., Gardasevic, J., \& Arifi, F. (2018c). Relationship between Foot Length Measurements and Body Height: A Prospective Regional Study among Adolescents in Central Region of Kosovo. Journal of Contemporary Medical Sciences, in press

Masanovic, B., Gardasevic, J., \& Arifi, F. (2018d). Standing Height and its Estimation Utilizing Foot Length Measurements in Adolescents from Southern Region in Kosovo. Sport Mont, 16(2), 27-31. doi: 10.26773/ smj.180605

Masanovic, B., Gardasevic, J., \& Arifi, F. (2018e). Sitting Height/Standing Height Relationship in Southern Region of Kosovo. Retrieved from SSRN's eLibrary: https://ssrn.com/abstract=3138523

Masanovic, B., Gardasevic, J., \& Arifi, F. (2018f). Sitting Height/Standing Height Relations in Central Region of Kosovo. Retrieved from SSRN's eLibrary: https://ssrn.com/abstract=3138525

Masanovic, B., Gardasevic, J., \& Arifi, F. (2018g). Sitting Height/Standing Height Relationship Measurements in Northern Region of Kosovo. Retrieved from SSRN's eLibrary: https://ssrn.com/abstract=3138526

Milašinovic, R., Gardasevic, J., \& Bjelica, D. (2017). Body heiht and its estimation utilizing arm span measurements in male adolescents from northern region in Montenegro. Acta Kinesiologica, 11(2) 75-80.

Milasinovic, R., Popovic, S., Matic, R., Gardasevic, J. \& Bjelica, D. (2016a). Body Height and its Estimation Utilizing Arm Span Measurements in Male Adolescents from Southern Region in Montenegro. Sport Mont, 14(2), 21-3.

Milasinovic, R., Popovic, S., Jaksic, D., Vasiljevic, I. \& Bjelica, D. (2016a) Stature and its Estimation Utilizing Arm Span Measurements in Feale Adolescents from Southern Region in Montenegro. Sport Mont, 14(3), $15-8$.

Milasinovic, R., Popovic, S., Jaksic, D., Vasiljevic, I. \& Bjelica, D. (2016b). Stature and its Estimation Utilizing Arm Span Measurements in Feale Adolescents from Southern Region in Montenegro. Sport Mont, 14(3), $15-8$.

Mohanty, S.P., Babu, S.S. \& Nair, N.S., (2001).The use of arm span as a predictor of height. A study of South Indian women. Journal of Orthopedics Surgery, 9(1), 19-23.

Pineau, J. C., Delamarche, P., \& Bozinovic, S. (2005). Average height of adolescents in the Dinaric Alps (in French). Comptes Rendus Biologies, 328(9), 841-6.

Popovic, S. (2017). Local Geographical Differences in Adult Body Height in Montenegro. Montenegrin Journal of Sports Science and Medicine, 6(1), 81-7.

Popovic, S., Arifi, F., \& Bjelica, D. (2017a). Standing Height and its Estimation Utilizing Foot Length Measurements in Kosovan Adults: National Survey. International Journal of Applied Exercise Physiology, 6(2), 1-7.

Popovic, S., \& Bjelica, D. (2017). Body Height and its Estimation Utilizing Foot 
Length Measurements in Kosovan Adults: National Survey. In Abstract Book of the Sport Science Conference AESA 2017 (2), Amol: Faculty of Sport Sciences, Shomal University; Asian Exercise and Sport Science Association.

Popovic, S. Gardasevic, J., Masanovic, B., Arifi, F., \& Bjelica, D. (2017b). Standing Height and its Estimation Utilizing Foot Length Measurements in Adolescents from Western Region in Kosovo. Sport Mont, 15(3), 3-7.

Popovic, S. (2016). Body Height and its Estimation Utilizing Arm Span Measurements in Montenegrin Adults: National Survey. In Book of Summaries of 11th FIEP European Congress "Anthropological Aspects of Sport, Physical Education and Recreation" (5-6), Banjaluka: University of Banjaluka, Faculty of Physical Education and Sport.

Popović, S., Bjelica, D., Milašinović, R., \& Gardašević, J. (2016). Body height and its estimation utilizing arm span measurements in male adolescents from northern region in Montenegro. In Book of Abstracts of 4th International Scientific Conference "Exercise and Quality of Life" (38), Novi Sad: Faculty of Sport and Physical Education.

Popovic, S., Milasinovic, R., Matić, R., Gardašević, J., Bjelica, D. (2016). Body height and its estimation utilizing arm span measurements in male adolescents from southern region in Montenegro. In Book of Abstracts of the 13th International Scientific Conference on Transformation Process in Sport "Sport Performance" (29-30), Podgorica: Montenegrin Sports Academy.

Popovic, S. \& Bjelica, D. (2016). Body Height and its Estimation Utilizing Arm Span Measurements in Kosovan Adolescence: National Survey. In Abstract Book of International Eurasian Conference on Sport, Education and Society (9), Antalya: International Science Culture and Sport Association.

Popovic, S., Bjelica, D., Tanase, G.D., \& Milasinovic, R. (2015). Body Height and Its Estimation Utilizing Arm Span Measurements in Bosnian and Herzegovinian Adults. Montenegrin Journal of Sports Science and Medicine, 4(1), 29-36.

Popovic, S., Bjelica, D., \& Hadzic, R. (2014a). Average body height of adolescents in Montenegro. In Proceedings book of the 13th International Sport Sciences Congress (462- 463). Konya: Selcuk University.

Popovic, S., Bjelica, D., Molnar, S., Jaksic, D., \& Akpinar, S. (2013). Body Height and Its Estimation Utilizing Arm Span Measurements in Serbian Adults. International Journal of Morphology, 31(1), 271-9.

Popovic, S., Bjelica, D., Petkovic, J.\& Muratovic, A. (2012). Comparative Study of Anthropometric Measurement and Body Composition between Elite Soccer and Handball Players. In Proceedings Book of the 4th International Scientific Conference "Contemporary Kinesiology"(102-108). Split: Faculty of Kinesiology, University of Split.

Reeves, S.L., Varakamin, C., \& Henry, C.J. (1996). The relationship between arm-span measurements and height with special reference to gender and ethnicity. European Journal of Clinical Nutrition, 50(6), 398-400.

Quanjer, P.H., Capderou, A., Mazocioglu, M.M., Aggarwal, A., Popovic, S. Datta Banik, S., Tayie, F.A. K., Golshan, M., Ip, M.S.M., \& Zelter, M. (2014) All-age relationship between arm span and height in different ethnic groups. European Respiratory Journal, 44, 905-12.

Steele, M.F., \& Chenier, T.C. (1990). Arm-span, height and age in black and white women. Annals of Human Biology, 17(6), 533-41.

Ter Goon, D., Toriola, A.T., Musa, D.I., \& Akusu, S. (2011). The relationship between a span and Body Height in Nigerian adults. Kinesiology, 43(1), 38-43.

Vujovic, D., Bubanja, M., Tanase, G. D. \& Milasinovic, R. (2015). Body Height and Its Estimation Utilizing Arm Span Measurements in Male Adolescents from Central Region in Montenegro. Sport Mont, 12(43-45), 283-8. 